\title{
Corrigendum: Lexical development of noun and predicate comprehension and production in isizulu
}

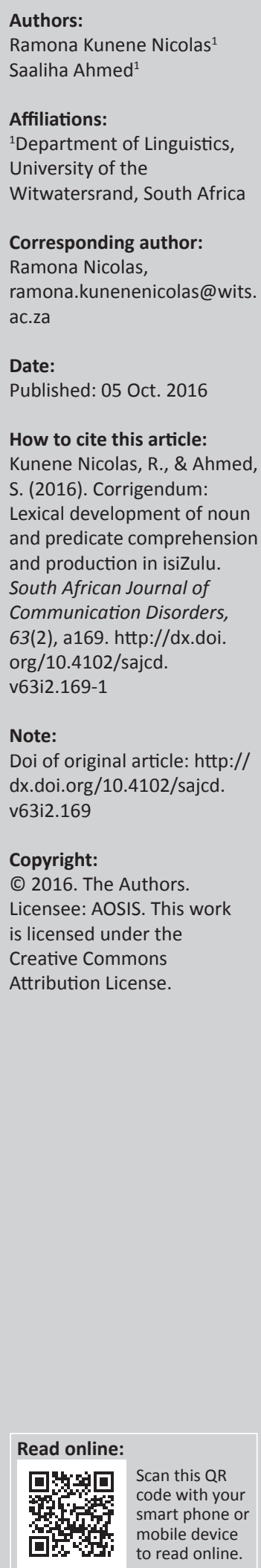

The authors apologise for omitting the following from their article, Lexical development of noun and predicate comprehension and production in isiZulu, published 28 July 2016:

- Introduction: adding additional text to the last paragraph, in order to explicitly name the project, which includes the URL of the project.

- Methods: adding additional text, acknowledging that the method is part of the joint project.

- Ethical considerations: text amended.

- Acknowledgements: including the project collaborators in their totality and the grant number of the funding received.

The above changes have been made to the article. For more information please see the updated sections, which reflects the full details as follows:

\section{Introduction}

This study seeks to investigate the development of noun and predicate comprehension (PC), and production in isiZulu-speaking children between the ages of 25 and 36 months. The study is part of an international research collaboration that aims to investigate speech and co-speech gesture production and comprehension development in children under the research project entitled 'FP7Marie Curie "Gesture and Language Development across Romance and Bantu languages" research programme (PIRSES-GA-2013-612563 GEST LAN D)' (http://www.gestland.eu). It compares lexical comprehension and production of two romance languages, Italian and French, and two South African Bantu languages, isiZulu and Sesotho, using an Italian developed and validated vocabulary assessment tool. This paper presents the preliminary findings of the lexical development of isiZulu speakers.

\section{Methods}

The data collection procedures, adaptation of PiNG, coding and analysis was a joint effort on the part of the four teams on the Gestland project. This paper only focuses on the adaptation to isiZulu.

\section{Stage 1: Translation of the PiNG lexicon into the target languages}

After the initial translation of the PiNG assessment tool from Italian by the Italian collaborators, a translation of the set of nouns (20 target nouns in the comprehension task +20 target nouns in the production task $+2 \times 2=4$ lexical items for the pre-tests) and the set of predicates (20 target verbs and/or adverbs and/or adjectives in the comprehension task +20 target verbs and/or adverbs and/or adjectives in the production task $+2 \times 2=4$ lexical items for the pre-tests) was carried out in isiZulu by the researcher, who is a native speaker of isiZulu and a linguist, together with two isiZulu-speaking research assistants, who are also linguists. The translation was further tested in a pilot study of native Zulu adults for validation (see Stage 2).

\section{Ethical considerations}

The pilot study as well as the main study followed ethical considerations. Children participants were selected after their parents had signed a consent form, which was written in English and isiZulu. Caregivers and parents were allowed to watch the administration of the PiNG assessment tool. Participant information was anonymised and children files were allocated anonymous file names in line with the project protocols. The Wits HREC Non-Medical Ethics Committee granted ethical clearance for the study (Protocol number H13/08/43). 


\section{Acknowledgements}

The research leading to these results has received funding from the European Community's Seventh Framework Programme (FP7/2007-2013) under Grant Agreement $\mathrm{n}^{\circ} 612563$. The programme is entitled 'FP7-Marie Curie "Gesture and Language Development across Romance and Bantu languages" research programme (PIRSES-GA2013-612563 GEST LAN D)'. The Gestland study team is comprised of the following members: Jean-Marc Colletta (PI), Ali Hadian Cefidekhanie, Elnaz Jalilian, University of Grenoble Alpes; Olga Capirci (Co-PI), Laura Sparaci, Francesca Lasorsa, Morgana Proietti, GLADD Lab, CNR, Rome; Heather Brookes (Co-PI) Thato Nteso, Mpho Semenye,
University of Cape Town; Ramona Kunene Nicolas (Co-PI), Gabby Dlamini, Saaliha Ahmed, Nonhlanhla Ntuli, University of the Witwatersrand, Johannesburg. We thank the research partners of the Gestland project: Jean-Marc Colletta, Olga Capirci and Heather Brookes. A special acknowledgement goes to Olga, for her assistance with the PiNG tool and scientific expertise on related literature. We thank the participating children, the staff from the crèches, and the parents and the caregivers who volunteered to participate in this study. We also thank the Wits team of researchers who assisted in the data collection: Nonhlanhla Ntuli and Karabo Ragumundo. We acknowledge the research assistants who coded this data: Saaliha Ahmed, Raheema Amiroodeen and Nonhlanhla Ntuli. 